\title{
Factor Xa inhibitors: critical considerations for clinical development and testing
}

\author{
Richard C. Becker ${ }^{1}$ (D)
}

Accepted: 13 April 2021 / Published online: 15 May 2021

(c) The Author(s), under exclusive licence to Springer Science+Business Media, LLC, part of Springer Nature 2021

\begin{abstract}
The selection of factor $(\mathrm{F}) \mathrm{X}$ and its activated protease FXa for targeted inhibition to prevent and treat thrombotic conditions is based on an understanding of coagulation biochemistry, sequential steps that occur on tissue factor bearing cells and the interface of coagulation proteins, platelets, mononuclear cells and the nuclear constituents of inflammatory cells. The goal for developing direct oral FXa inhibitors was to achieve rapid, selective, predictable, safe and effective anticoagulation across a broad group of patients expected to derive benefit. The history and development in patient care are exemplars of knowledge, translation and collaboration between the public and private sectors.
\end{abstract}

Keywords Factor X $\cdot$ Direct oral anticoagulants $\cdot$ Direct factor Xa inhibitors

\section{Highlights}

- Direct oral anticoagulants are the end-result of decades of collaboration among, scientists, clinical researchers, practicing clinicians, regulators and the lay community.

- Reversal and replacement strategies to mitigate bleeding risk have evolved, however, further investigation is needed.

\section{Laying the foundation for direct oral factor Xa inhibitors}

The waterfall cascade of coagulation [1] and the cell based model of coagulation are two complementary underpinnings for factor (F) X (FX) and its activated protease, FXa as a target for pharmacological inhibition [2]. The former is an example of an orderly sequence of events that fulfills fundamental rules of protein biochemistry in physiological systems. Specifically, shape and three-dimensional structure determine function [3]. The latter captures the environment that is necessary and sufficient for production of a

Richard C. Becker

Richard.becker@uc.edu

1 University of Cincinnati Heart, Lung and Vascular Institute, 231 Albert Sabin Way, Cincinnati, OH 45267, USA product following completion of one or more biochemical reactions. Thrombin is the pivotal final product of coagulation protein assembly and activation on tissue factorbearing cells that characterize physiological hemostasis and pathological thrombosis [4]. The one exception to this rule in physiological coagulation is the contact activation system that responds to non-biological conditions, including foreign surfaces, infectious pathogens and constituents of a cell nucleus (nucleic acids, histones, and chromatin) released under extreme conditions [5] (Fig. 1).

\section{Early discussions: sharing a common goal}

Clinicians, pharmacists and researchers trained in the past half-century are familiar with oral anticoagulants and their use in patient care. Less than two-decades ago, they had only one drug, warfarin, a racemic mixture of two active enantiomers- R- and S-forms with complex pharmacokinetics, pharmacodynamics, drug-food interactions, drug-drug interactions and a very narrow therapeutic window (for efficacy and safety). The complexity of warfarin in patient care that began with initial approval for medical use in 1954 $[6,7]$ was amplified by its variable dose-response effects requiring the development of a specific test, the international normalized ratio (INR) and individual patient dose-titration [8]. Warfarin stands alone as a drug not administered in fixed doses across patients with a common indication for 
Fig. 1 A cell-based model of coagulation has three distinct phases: initiation, amplification and propagation. The initiation phase begins on tissue-factor bearing cells that include injured endothelial cells, monocytes, macrophages and transformed smooth muscle cell among others. Assembly of the tenase complex is sufficient to generate a small amount of thrombin, activate platelets and amplify the production of thrombin that propagates the assembly of coagulation proteins on aggregated platelets with an increasingly large surface area. From Hoffman M. Thromb and Haemostas 2001; 85: 958-965. With permission

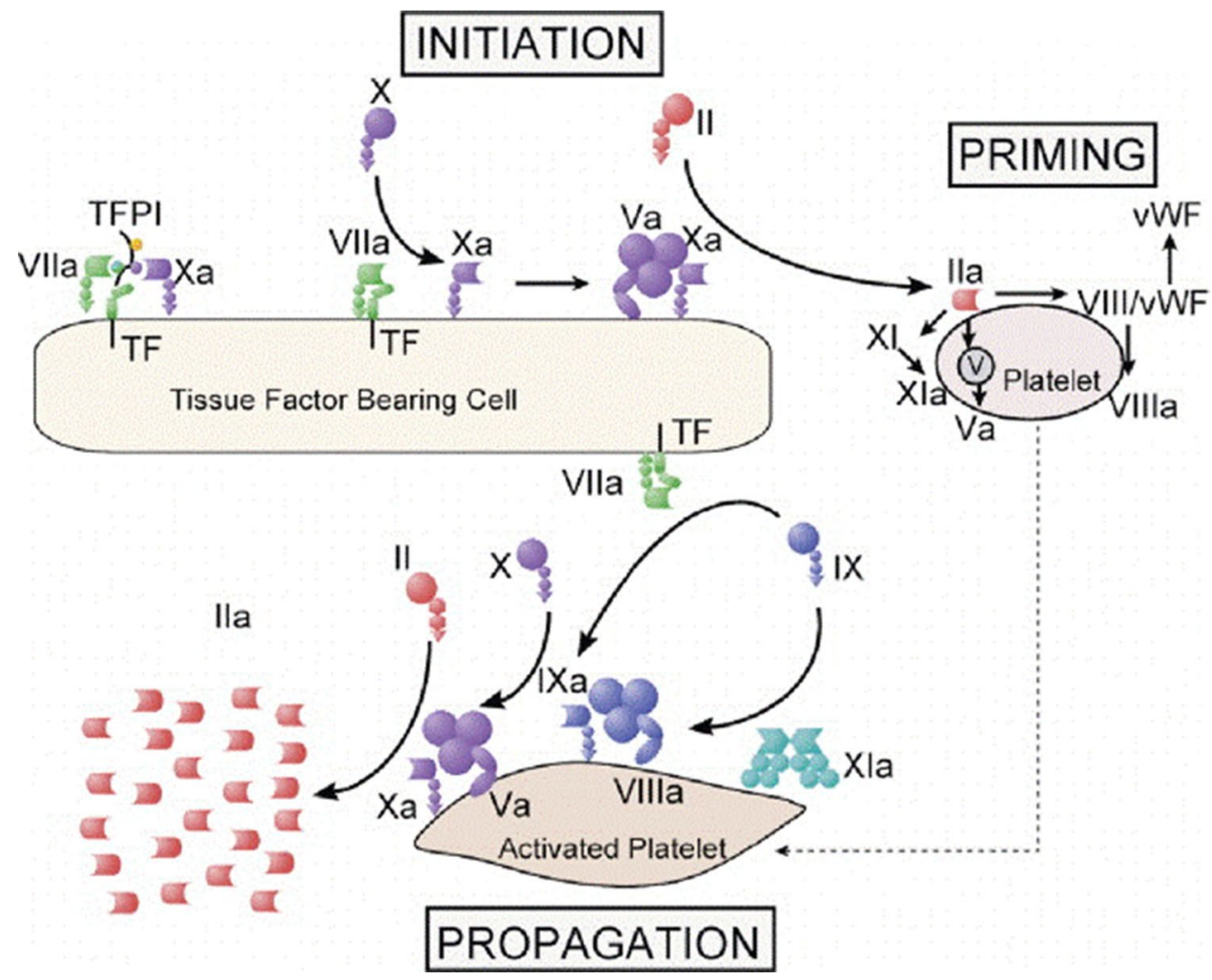

anticoagulant therapy that requires routine monitoring by blood testing to achieve a "therapeutic level", and classified as an "extremely hazardous substance". The last unique designation for a drug used to treat human disease derives from Sect. 302 of the United States Emergency Planning and Community Right-to-Know Act (EPCRA) — a federal law passed by the 99th congress (United States Environmental Protection Agency. epa.gov/epcra-a accessed November 22, 2020). Warfarin's complexity fostered the creation of dedication clinics and health care infrastructures to educate patients and providers, develop dose-titration algorithms and integrate specially trained providers (pharmacists, nurses, nurse practitioners, physicians, and technicians) to better achieve optimal outcomes [9].

Need for an oral anticoagulant or class of anticoagulants that offered predictable pharmacokinetics and pharmacodynamics, few food-drug or drug-drug interactions, fixed dosing options, no need for routine monitoring and a broader therapeutic window than warfarin was voiced equally by the medical, scientific, pharmaceutical, federal and regulatory communities. The end-result of round-table discussions, workshops, think tanks, forums, colloquia, conferences, seminars, market research, stakeholder mapping and analysis and logical reasoning was a robust, global drug development initiative. An equally robust collaboration between the public and private sectors led to the development of a new class of direct oral factor IIa and Xa inhibitors. Phase 1, 2, and 3 clinical trials of patients with and those at risk for
Table 1 Clinical Indications for direct oral factor X inhibitors

\begin{tabular}{|c|c|c|}
\hline Drug & Initial FDA approval & Approved clinical indications \\
\hline Rivaroxaban & November 4, 2011 & $\begin{array}{l}\text { AF } \\
\text { VTE prevention }{ }^{+, \#} \\
\text { VTE Treatment } * *^{*} \\
\text { VTE Treatment-extended } \\
\text { CCAD } \\
\text { PAD }\end{array}$ \\
\hline Apixaban & December 28, 2012 & $\begin{array}{l}\text { AF } \\
\text { VTE Prevention }{ }^{+} \\
\text {VTE treatment }{ }^{* *}\end{array}$ \\
\hline Endoxaban & June 23, 2017 & $\begin{array}{l}\text { AF } \\
\text { VTE Treatment }^{++}\end{array}$ \\
\hline Betrixaban & January 8, 2015 & VTE Prevention ${ }^{\#}$ \\
\hline
\end{tabular}

$A F$ atrial fibrillation, $V T E$ venous thromboembolism, $C C A D$ chronic coronary artery disease, $P A D$ peripheral arterial disease

*As of December 1, 2010

${ }^{+}$Following hip or knee replacement surgery

**Deep vein thrombosis and pulmonary embolism

${ }^{++}$In patients already treated with parenteral anticoagulants for 5-10 days

\# In adult patients hospitalized for an acute medical illness at risk for thromboembolic events

${ }^{\mathrm{O}}$ For patients at risk for recurrent VTE after completing 6 months of treatment 
Table 2 Pharmacokinetic properties and dosing for the direct oral factor Xa inhibitors

\begin{tabular}{lllll}
\hline & Rivaroxaban & Apixaban & Betrixaban & Edoxaban \\
\hline Bioavailability & $>80 \%$ & $>50 \%$ & $\sim 35 \%$ & $>80 \%$ \\
Onset of action, hrs & $2-4$ & $\sim 3$ & $1-3$ & $1-2$ \\
Half-life, hrs & $5-13$ & $9-14$ & $\sim 20$ & $8-10$ \\
Metabolism & $1 / 3$ renal; 2/3 liver & Multiple pathways & Via bile ( 5\% renal) & Multiple (majority renal) \\
& (CYP 450) & $(25 \%$ renal) & & Low-Mod \\
Likehood of drug interactions & Low-Mod & Low & Low & Oral once daily \\
Dosing & Oral once daily & Oral twice daily & Oral once daily & \\
\hline
\end{tabular}

thrombosis of the venous (deep vein thrombosis, pulmonary embolism) and arterial (atrial fibrillation, stroke, and chronic coronary syndrome) circulatory systems were undertaken [10-13]. An extraordinary amount of data were generated and carefully analyzed to determine optimal dosing, safety profiles, patient selection, sustainable effects and cost-effectiveness. While the widespread interest to develop alternatives to warfarin in the form of direct oral anticoagulants (DOACs) produced dozens of drug candidates in the 1990s and early 2000s, ultimately four drugs emerged-rivaroxaban, apixaban, edoxaban and betrixaban (Tables 1 and 2).

\section{Direct oral factor $\mathrm{X}$ inhibitors}

\section{Fundamental properties}

The direct oral factor $\mathrm{Xa}$ anticoagulants bind in an L-shaped fashion to factor Xa's active site (reviewed in
Steinberg and Becker) [14]. The 'L' configuration includes the S1 and S4 binding sites of factor Xa. At these ends of the 'L', natural compounds possess polar, charged components, allowing them to bind the target specificity. Synthetic inhibitors instead possess aromatic rings with various moieties attached. These allow for alternative interactions in the S1 and S4 pocket, maintaining binding strength while maximizing bioavailability. Instead of polar ionic interactions, several of the direct oral factor $\mathrm{Xa}$ inhibitors are dependent on hydrophobic and hydrogenbonding interactions with the target.

Target-binding specificity directly associates with specific structures of these molecules (Fig. 2). Various substitutions of S1-pocket binding groups, from chlorine to fluorine, or other carbon-based groups, demonstrated significant variability in inhibitor activity [15]. However, several optionsmaintained target specificity, with a variety of different properties.
Fig. 2 Conceptual diagrammatic structures of the four FDA-approved direct oral factor Xa inhibitors binding to the active site. Structures for rivaroxaban and apixaban are derived from known structures, while betrixaban and edoxaban are extrapolated based on these structures. Not drawn to scale. $S 1$ and $S 4$ represent binding regions for the factor Xa active site; $O x$ oxazolidinone moiety, $\mathrm{Cl}$ chloride, $H$ hydrogen participating in bonding, $\mathrm{CH}_{3}$ methyl group, $\mathrm{H}_{2} \mathrm{O}$ water, $\mathrm{Tyr}$ tyrosine, Gly glycine, Gln glutamine, FDA (Food and Drug Administration). From Steinberg B and Becker RC. J Thromb Thrombolysis 2014; 37: 234-241. With permission
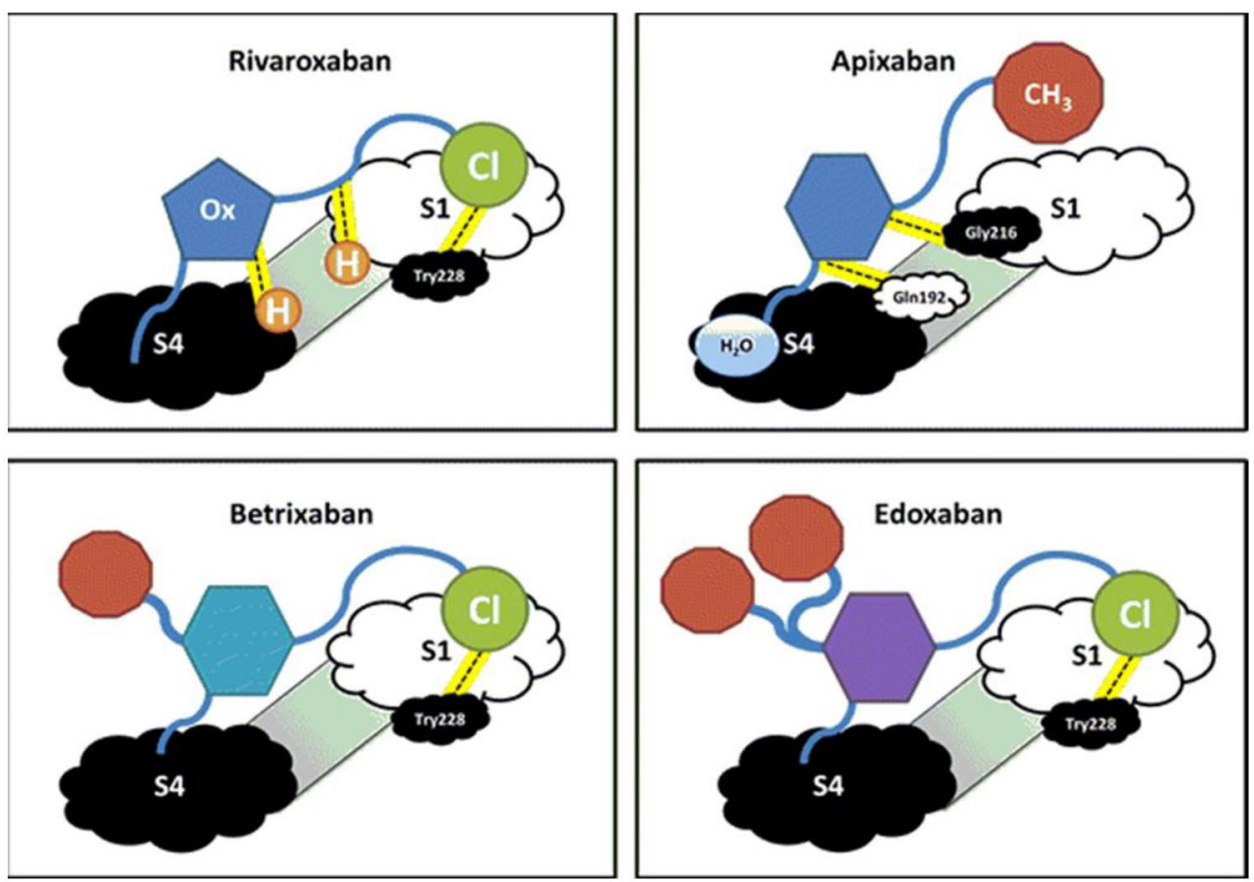


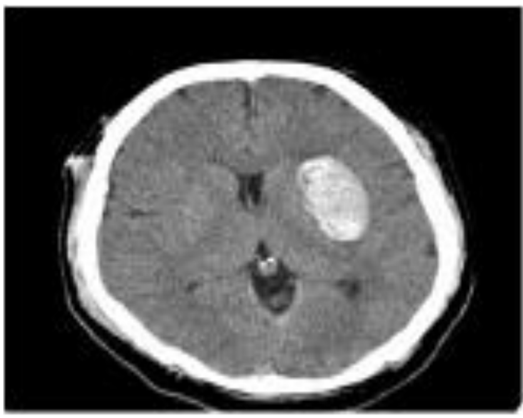

A

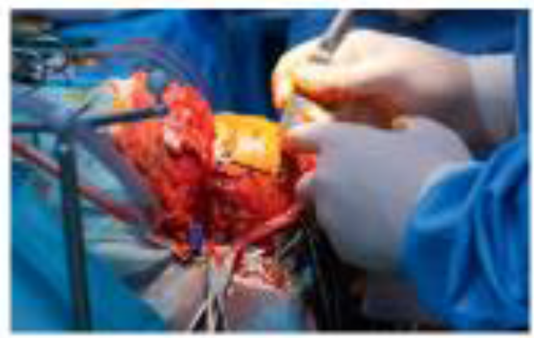

D

Fig. 3 Anticoagulant therapy is associated with a risk for spontaneous and provoked bleeding that can be serious or life threatening. Panel A- a spontaneous intraparenchymal bleed; panel B- a gastrointestinal bleed in a patient with peptic ulcer disease; panel C- an intraperitoneal bleed in a patient who was in a motor vehicle accident;

\section{The importance of drug reversibility}

A common theme in early discussions of DOACs was drug reversibility. This was not a surprise based on an existing precedent for warfarin and the potential risk for bleeding to include serious, life-threatening and life-altering events (Fig. 3). Moreover, clinicians had strived for years to balance the need for anticoagulation to reduce thrombotic and thromboembolic events with hemostasis in patients undergoing procedures and surgery associated with heightened bleeding risk. The desire to have the best of both worlds played out in the form of clinical trials and numerous guidance documents for bridging anticoagulant therapy [16-18]. The lay and clinical communities reached a place of confidence through best practice, meticulous monitoring and the wide-scale availability of tested and validated strategies to reverse warfarin's anticoagulant effect [19, 20].

Experienced clinicians recognized that vitamin $\mathrm{K}$ was not a warfarin antidote and plasma-derived blood products, or recombinant coagulation factors were replacement products rather than reversal agents. They also knew that the primary goals of treatment were to generate thrombin, over-ride systemic anticoagulant effects and achieve panel D- a intracranial operation requires normal hemostasis as does panel-E spinal surgery. The urgency of invasive procedures associated with bleeding risk determines the need for reversal agents and the timing of their administration

hemostatic stabilization while vitamin K- dependent coagulation proteins entered the circulation.

The clinical stakeholders expected nothing less for DOACs. The clinical trial experts tasked to design and conduct phase 2 and phase 3 registration trials with a clear goal of protecting study participants, stood shoulder-to-shoulder with their clinician colleagues and advisors. Ultimately, strategies and algorithms for replacement or repletion of coagulation proteins were developed and put in place (to the relief of consenting participants and site investigators and research coordinators alike). The fact remained that antidotes and targeted reversal agents were not available nor were there plans for concomitant development with any of the DOACs.

\section{What changed the conversation?}

The drive for DOAC reversal agents came to the forefront and steadily mounted soon after the Food and Drug Administration approval and wide-scale prescribing [21, 22]. Despite a lower or similar risk for major bleeding when compared with warfarin titrated to a known therapeutic (safe and effective) INR [23, 24], and marked reduction $(\sim 70 \%)$ for intracranial bleeding across the entire class of new anticoagulants, 
prescribing clinicians and their patients initially took pause for three reasons. First, the cost of treatment, including high co-payments. Second, a lack of familiarity (not a surprise given more than a one-half century experience with warfarin as the only oral anticoagulant). Third, the lack of a reversal agent. A combination of factors, including market forces led to the development of reversal agents [25] and management guidelines [26] for patients with and those at risk for DOAC-associated bleeding complications. There are opportunities for improvement and further development of reversal agents [27].

\section{Clinical gaps and unmet needs}

DOACs in general and direct oral FXa inhibitors in particular have improved the care of patients with thrombotic disease, disorders and conditions. The road to achieving optimal outcomes begins with several fundamental factors, including drug availability, affordability, indicationspecific utilization and implementation consistency. The availability of generic formulations may favorably lessen cost- related barriers (United States Food and Drug Administration; fda.gov; accessed November 26, 2020).

The safe and effective use of direct oral FX inhibitors in elderly patients [28], those with advanced renal insufficiency, bioprosthetic [29] and mechanical heart valves [30], transcatheter aortic valve replacement [31], cancer (thrombosis prevention and treatment), and specific ethnic background that might affect metabolism, clearance and overall risk for anticoagulant therapy will require further investigation. Similarly, their role in the management of patients with COVID-19 is the focus of several large clinical trials (Accelerating COVID-19 Therapeutic Interventions and Vaccines (ACTIV-4)) supported by the National Institutes of Health, National Heart, Lung and Blood Institute and other federal agencies working collaboratively with the academic community and pharmaceutical industry (nih.gov; accessed November 26, 2020).

\section{One size does not fit all}

Quantitative systems pharmacology (QSP), defined as the approach to translational medicine that combines computational and experimental methods to elucidate, validate and apply new pharmacological concepts to the development and use of small molecule and biologic drugs acknowledges that one size does not fit all in optimal treatment. QSP provides an integrated "systems level" approach to determining mechanisms of action of new drugs in preclinical and animal models and in patients [32]. The complex cellular and protein networks in coagulation, coupled with individual human physiology in health and disease provides the backdrop for maximizing therapeutic benefit and minimizing toxicity in "precision medicine" [33]. Existing databases of direct oral factor Xa inhibitors provide the foundation for an in-depth evaluation and for building quantitative model platforms to improve our mechanistic understanding of drug treatment at a patient-specific level. These models may, in turn, reveal significant components from the multi- dimensional data sets and expand our current understanding of one or more networks controlling the regulation and effect of FXa inhibition. This approach does not replace the potential role of current laboratory assays that might be useful in specific clinical scenarios such as risk assessment before surgery with anticipated bleeding or following reversal or replacement therapy [34], but rather to expand the field's ability to capture bidirectional interactions between pharmacotherapy and disease states.

\section{Conclusion and future directions}

The development of direct oral factor Xa inhibitors followed decades of scientific investigation and an understanding of physiological hemostasis and pathological thrombosis. The prevention and treatment of thrombotic conditions involving the venous and arterial circulation represents the result of collaborative undertakings by the academic, pharmaceutical and lay communities striving to improve patient care. There have been advances, but opportunities remain to reach a greater proportion of patients in need, apply precision medicine at the ground level, and improve upon reversal agents and strategies in contemporary patient care.

\section{References}

1. Hougie C (2006) A review of the scientific and literary accomplishments of Professor R. G. Macfarlane CBE. FRS. Br J Haematol 133(6):581-590

2. Hoffman M, Monroe DM 3rd (2001) A cell-based model of hemostasis. Thromb Haemost 85(6):958-965

3. Doktorova M, Symons JL, Levental I (2020) Structural and functional consequences of reversible lipid asymmetry in living membranes. Nat Chem Biol 16(12):1321-1330

4. Becker RC (2006) Biosignatures in thrombotic disorders. Circulation 22(2): 157-158

5. Woodruff RS, Sullenger B, Becker RC (2011) The many faces of the contact pathway and their role in thrombosis. J Thromb Thrombolysis 32(1):9-20

6. Clatanoff DV, Triggs PO, Meyer OO (1954) Clinical experience with coumarin anticoagulants warfarin and warfarin sodium. AMA Arch Intern Med 94(2):213-220

7. Shapiro S (1953) Warfarin sodium derivative: (coumadin sodium); an intravenous hypoprothrombinemia-inducing agent. Angiology 4(4):380-390 
8. Poller L (2004) International Normalized Ratios (INR): the first 20 years. J Thromb Haemost 2(6):849-860

9. Ellis RF, Stephens MA, Sharp GB (1992) Evaluation of a pharmacy-managed warfarin-monitoring service to coordinate inpatient and outpatient therapy. Am J Hosp Pharm 49(2):387-394

10. Varnes BE, Singh V, May CC (2021) Management of warfarin-associated intracerebral hemorrage before and after implementation of an order set and prospective pharmacist order verification. J Thromb Thrombolysis. https://doi.org/10.1007/ s11239-021-02404-8

11. Kearon C, Hirsh J (1997) Management of anticoagulation before and after elective surgery. N Engl J Med 336(21):1506-1511

12. Ansell JE (2000) The quality of anticoagulation management. Arch Intern Med 160(7):895-896

13. Turpie AG (2007) Oral, direct factor Xa inhibitors in development for the prevention and treatment of thromboembolic diseases. Arterioscler Thromb Vasc Biol 27(6):1238-1247

14. Steinberg BA, Becker RC (2014) Structure-function relationships of factor Xa inhibitors: implications for the practicing clinician. $\mathrm{J}$ Thromb Thrombolysis 37(2):234-241

15. Corte JR et al (2008) Structure-activity relationships of anthranilamide-based factor Xa inhibitors containing piperidinone and pyridinone P4 moieties. Bioorg Med Chem Lett 18(9):2845-2849

16. Serhal M, Barnes GD (2019) Venous thromboembolism: a clinician update. Vasc Med 24(2):122-131

17. Barnes GD, Kanthi Y, Froehlich JB (2015) Venous thromboembolism: predicting recurrence and the need for extended anticoagulation. Vasc Med 20(2):143-152

18. Douketis JD et al (2015) Perioperative bridging anticoagulation in patients with atrial fibrillation. N Engl J Med 373(9):823-833

19. Elsamadisi $P$ et al (2020) Weight-based dosing versus a fixed-dose regimen of 4-factor prothrombin complex concentrate in obese patients requiring vitamin $\mathrm{k}$ antagonist reversal. Am J Cardiovasc Drugs. https://doi.org/10.1007/s40256-020-00442-w

20. Bitonti MT et al (2020) Prospective evaluation of a fixed-dose 4-factor prothrombin complex concentrate protocol for urgent vitamin k antagonist reversal. J Emerg Med 58(2):324-329

21. Chaudhary R et al (2020) Direct oral anticoagulants: a review on the current role and scope of reversal agents. J Thromb Thrombolysis 49(2):271-286

22. Huisman MV, Fanikos J (2016) Idarucizumab and factor Xa reversal agents: role in hospital guidelines and protocols. Am J Med 129(11s):S89-s96
23. Patel MR et al (2011) Rivaroxaban versus warfarin in nonvalvular atrial fibrillation. N Engl J Med 365(10):883-891

24. Giugliano RP et al (2013) Edoxaban versus warfarin in patients with atrial fibrillation. N Engl J Med 369(22):2093-2104

25. Rivera-Caravaca JM et al (2020) Treatment strategies for patients with atrial fibrillation and anticoagulant-associated intracranial hemorrhage: an overview of the pharmacotherapy. Expert Opin Pharmacother 21(15):1867-1881

26. Kaide CG, Gulseth MP (2020) Current strategies for the management of bleeding associated with direct oral anticoagulants and a review of investigational reversal agents. J Emerg Med 58(2):217-233

27. Connolly SJ et al (2019) Full study report of andexanet alfa for bleeding associated with factor Xa inhibitors. N Engl J Med 380(14):1326-1335

28. Okumura K et al (2020) Low-dose edoxaban in very elderly patients with atrial fibrillation. N Engl J Med 383(18):1735-1745

29. Guimarães HP et al (2020) Rivaroxaban in patients with atrial fibrillation and a bioprosthetic mitral valve. N Engl J Med 383(22):2117-2126

30. Jawitz OK et al (2020) Rationale and design of PROACT Xa: A randomized, multicenter, open-label, clinical trial to evaluate the efficacy and safety of apixaban versus warfarin in patients with a mechanical On-X Aortic Heart Valve. Am Heart J 227:91-99

31. Dangas GD et al (2020) A controlled trial of rivaroxaban after transcatheter aortic-valve replacement. N Engl J Med 382(2):120-129

32. Geerts H et al (2020) Quantitative systems pharmacology for neuroscience drug discovery and development: current status, opportunities, and challenges. CPT Pharmacometrics Syst Pharmacol 9(1):5-20

33. Irurzun-Arana I et al (2020) Beyond deterministic models in drug discovery and development. Trends Pharmacol Sci 41(11):882-895

34. Sarode R (2019) Direct oral anticoagulant monitoring: what laboratory tests are available to guide us? Hematol Am Soc Hematol Educ Program 2019(1):194-197

Publisher's Note Springer Nature remains neutral with regard to jurisdictional claims in published maps and institutional affiliations. 